\title{
Heartbeat: Focus on the Fontan patient
}

Effective surgical palliation for complex congenital heart disease in infancy and childhood allows these patients to live into adulthood but may be associated with adverse outcomes over the longer term. Management of adult patients with "Fontan physiology" is especially challenging. The Fontan operation was first described in 1971 so that the oldest surviving patients with this procedure are only in their 40's, with most being much younger.The physiology of the Fontan circulation, as elucidated in an elegant review by Gewillig and Brown (see page 1081), is characterized by an obligatory increase in systemic venous pressures because blood flow is directed to the pulmonary circuit without an intervening pumping chamber (e.g. absence of a functional right ventricle). In addition, forward cardiac output is lower than normal and is not responsive to normal physiological stressors, such as exercise (figure 1). In addition, over the long term, the Fontan may "fail" with an inadequate cardiac output to meet metabolic demands even at rest due to increasing pulmonary vascular resistance over time, ventricular dysfunction or refractory arrhythmias, as well as the effects of persistently elevated systemic venous pressure.

Over the past 25 year, the Fontan procedure was performed in over 1300 patients in Australia and New Zealand. Using this database, Shi and colleagues (see page 1120) found that freedom from Fontan failure was $74 \% \pm 3.9 \%$ at 20 years. A total of only 34 patients underwent heart transplantation (HTX) and there was significant regional variation in referral for HTX, even though outcomes after HTX in Fontan patients were similar to patients with other types of congenital heart disease (figure 2).

In another study of outcomes in Fontan patients (see page 1127), a retrospective analysis of surgical outcomes in 225 patients from 22 medical centers found that after Fontan completion, takedown was the most common procedure in the early postoperative phase whereas late

\section{Correspondence to}

Professor Catherine M Otto, Division of Cardiology, University of Washington, Seattle, WA 98195, USA; cmotto@u.washington.edu

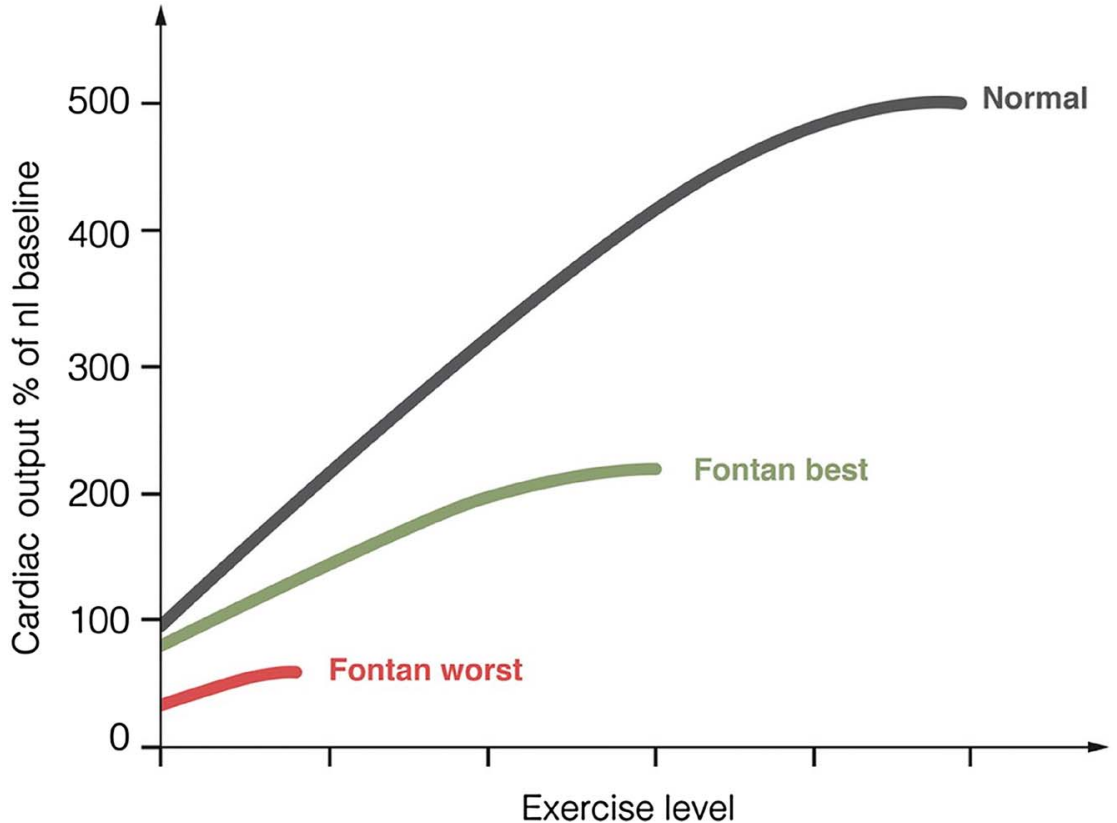

Figure 1 Exercise and output: normal versus Fontan circulation. Normal subjects with a biventricular circulation can increase their cardiac output up to five times (black line). At rest, patients with Fontan circulation at best already have a cardiac output $80 \%$ of normal and with a markedly restricted ability to increase during exercise (green line) allowing only a mild sporting ability. At worst (red line), the output is severely restricted at rest and barely increases during exercise. Adapted with permission from Gewillig and Goldberg. Heart Fail Clin 2014; 10: 105-16.

failure (most often manifested as arrhythmias) was treated with Fontan conversion or HTX. At a median follow-up of 5.9 (range 0 to 23.7 ) years, death or HTX occurred in $44.7 \%$ of the 38 Fontan takedown patients, $26.3 \%$ of the 137 Fontan conversion patients and $34.0 \%$ of the 50 HTX patients (figure 3).

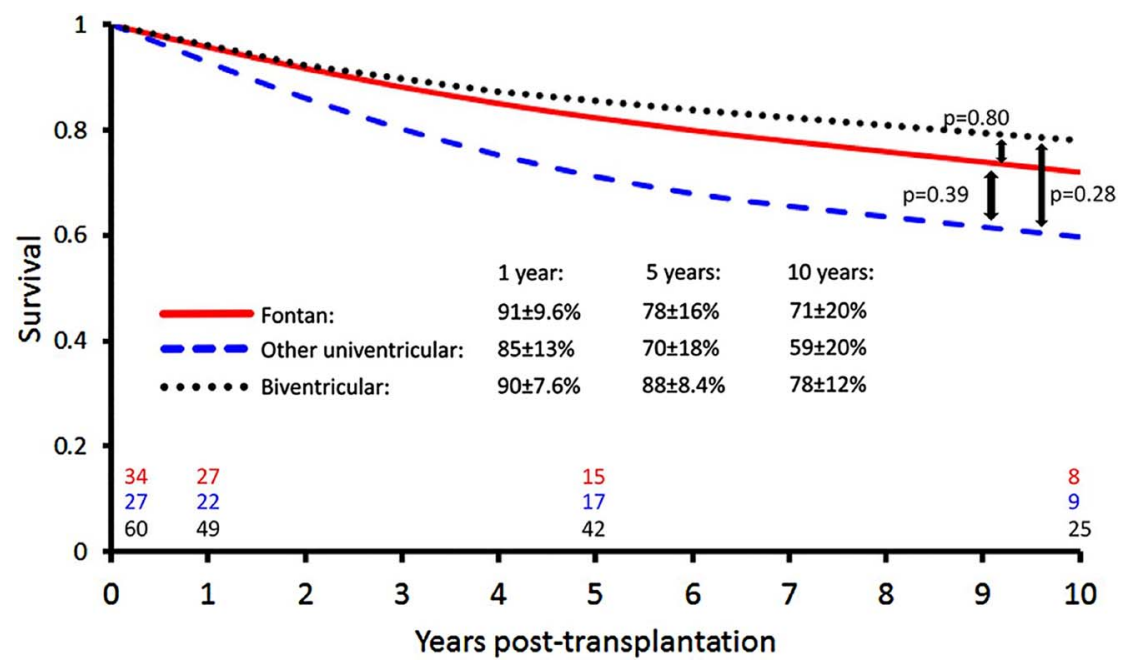

Figure 2 Survival after heart transplantation in Fontan patients compared and patients with other forms of congenital heart disease.

In an editorial, Backer (see page 1077) concludes: 'The high mortality for Fontan takedown reminds us to carefully select our patients for the Fontan operation. The decision of when to proceed with Fontan conversion versus heart transplantation is heavily based on the preservation of ventricular function. Patients with ventricular 


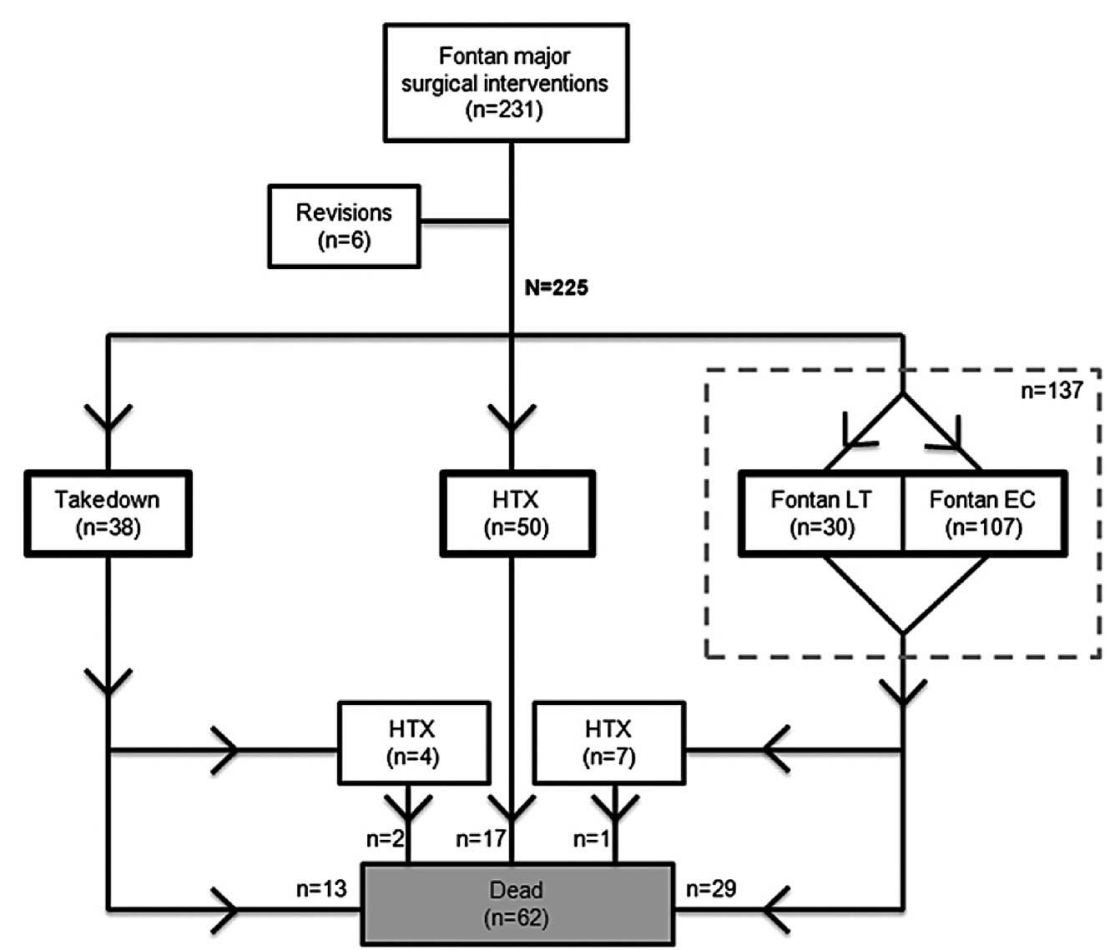

Figure 3 Flowchart describing the surgical treatment for patients with failing Fontan $(n=231)$. HTX, heart transplantation; LT, lateral tunnel; EC, extracardiac conduit.

dysfunction should be directed to heart transplantation". "It is also critically important that these complex patients be treated at institutions with the experience and expertise to achieve the best possible outcomes in a group of critically ill complex congenital heart patients".

This issue also included articles on occult diastolic dysfunction late after the Fontan procedure (see page 1109) as well as a systematic review of protein-losing enteropathy in Fontan patients (see page 1115).

Cardiologists are increasingly interested in integration of palliative care into management of patients with heart disease. Although we have made great strides in decreasing mortality and reducing morbidity due to heart failure, there has been less attention on improving quality of life in patients with end-stage heart failure (ESHF) refractory to treatment. Wong and colleagues (see page 1100) randomized 84 patients with ESHF to a transitional palliative care model compared to standard care. The primary outcome of hospital readmis-

Table 1 Readmission at 4 and 12 weeks

\begin{tabular}{|c|c|c|c|}
\hline & $\begin{array}{l}\text { Control } \\
(n=41)\end{array}$ & $\begin{array}{l}\text { Intervention } \\
(n=43)\end{array}$ & p Value \\
\hline Number of readmissions at 4 weeks (mean, SE) & $0.41(0.10)$ & $0.21(0.07)$ & 0.10 \\
\hline Number of readmissions at 12 weeks (mean, SE) ${ }^{* *}$ & $1.10(0.16)$ & $0.42(0.10)$ & 0.001 \\
\hline \multicolumn{4}{|l|}{ Readmissions within 28 days (n, \%) } \\
\hline No & $29(70.7 \%)$ & $34(79.1 \%)$ & 0.38 \\
\hline Yes & $12(29.3 \%)$ & $9(20.9 \%)$ & \\
\hline \multicolumn{4}{|l|}{ Readmissions within 84 days $(n, \%)^{*}$} \\
\hline No & $16(39.0 \%)$ & $29(67.4 \%)$ & 0.009 \\
\hline Yes & $25(61.0 \%)$ & $14(33.6 \%)$ & \\
\hline
\end{tabular}

sion at 12 weeks was lower in the palliative care group (33.6\%) compared to standard care $(61.0 \%, \mathrm{p}=0.009)$ with a relative risk for readmission of 0.55 (0.35 to 0.88). (table 1) In addition, palliative care resulted in significant improvements in depression, dyspnea and overall quality of life.

In the accompanying editorial, Kavalieratos, Rollman and Arnold (see page 1079) point out the potential social and medical differences between the study group in Hong Kong and ESHF patients in other countries. In addition, "Given that earlier receipt of palliative care is associated with greater benefit, we suggest that the transitional palliative care for ESHF be rebranded as an intervention for patients with 'advanced' heart failure instead of 'end-stage' heart failure, prioritising patient need over prognosis". "In order for palliative care to be most effective, it needs to be divorced from false opposition of curative versus (not and) palliative care". Currently, there are many barriers to integrating palliative care approaches into our routine management of heart failure patients; the emerging evidence suggests it is time for us to work to overcome those barriers.

The Education in Heart article (see page 1142 ) in this issue discusses gender differences in patients with coronary artery disease. This article summarizes the evidence that women have "atypical" symptoms compared to men (although maybe it is the men who are "atypical") and that women have worse outcomes, possibly related to comorbidities, in addition to under-treatment. Perhaps it is time to move from describing "differences" to ensuring equity in medical care for coronary disease in women as well as men.

The Image Challenge in this issue (see page 1126) shows an unexpected finding on coronary angiography in an 80 year old man who presented with chest pain. See if you can make the correct diagnosis!

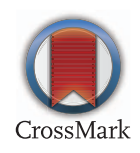

To cite Otto CM. Heart 2016;102:1073-1074.

Heart 2016;102:1073-1074.

doi:10.1136/heartjnl-2016-310114 